\title{
Multiple Confluent Telangiectatic Lesions Following Radiation Treatment for Prostate Cancer
}

\section{Y. Komuro, T. Watanabe, H. Nagawa} Department of Surgical Oncology, Faculty of Medicine, University of Tokyo, Tokyo, Japan

\section{Corresponding Author}

\section{Y. Komuro, M.D.}

Department of Surgical Oncology Faculty of Medicine University of Tokyo

7-3-1 Hongo

Bunkyo-ku

Tokyo 113-8655

Japan

Fax: $\quad+81-3-38116822$

E-mail: komuro@kt.rim.or.jp

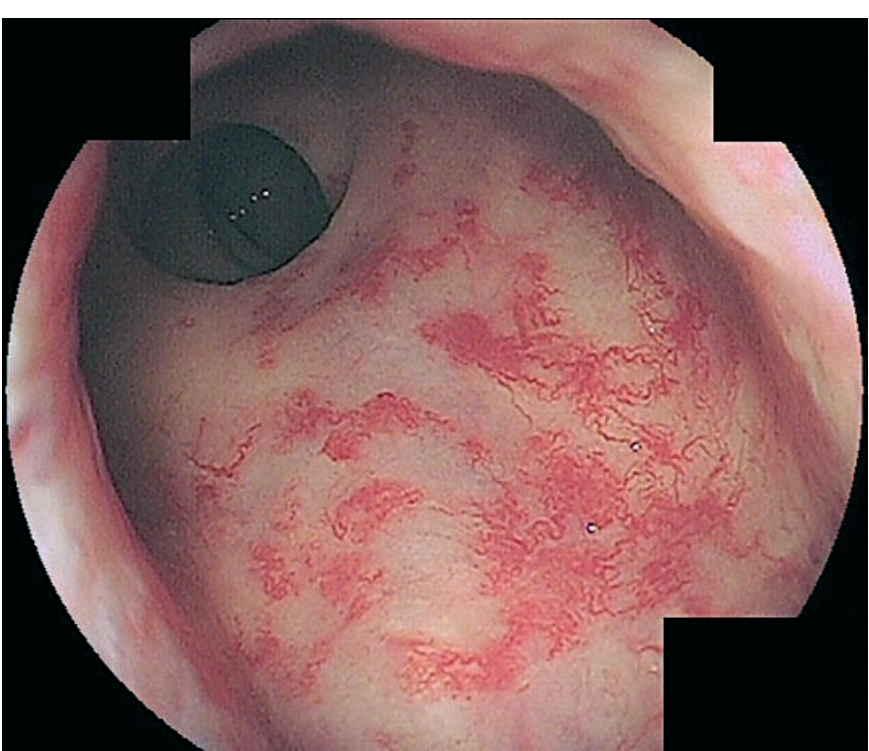

Figure 1 Endoscopic view of chronic radiation-induced proctitis. The anterior rectal mucosa appeared edematous and friable with multiple confluent neovascular telangiectatic lesions. The patient had been treated 3 years previously for prostate cancer, with a total dose of 60 Gy. 